SiF 2020 - The $11^{\text {th }}$ International Conference on Structures in Fire

The University of Queensland, Brisbane, Australia, 30th November - 2nd December, 2020

\title{
PROBABILISTIC MODELS FOR THERMAL PROPERTIES OF CONCRETE
}

\author{
Balša Jovanović $^{1}$, Negar Elhami Khorasani ${ }^{2}$, Thomas Thienpont ${ }^{3}$, Ranjit Kumar Chaudhary ${ }^{4}$, Ruben Van \\ Coile ${ }^{5}$
}

\begin{abstract}
Thermal conductivity and specific heat of concrete are highly influential parameters for the heat transfer into the material during fire exposure. Reviewing the available literature has shown that there is a large scatter in the data for these thermal parameters. To quantify that uncertainty, novel probabilistic models for thermal conductivity and specific heat of concrete at elevated temperatures are developed.

Analysis of available experimental data indicates that a temperature-dependent Gamma distribution can be recommended for both thermal properties. Closed-form equations for the temperature-dependent mean and standard deviation are derived. Thus, for both the thermal conductivity and the specific heat, a continuous probability distribution as a function of temperature is obtained, which can be easily implemented in numerical simulations. Using the example of the probabilistic analysis of a simply supported concrete slab exposed to the standard fire, the models are compared with the commonly used deterministic representation of the thermal properties. It is shown that the calculated probabilities of failure using the deterministic models are an order of magnitude lower and therefore unconservative. This analysis suggests that accounting for the uncertainty in thermal properties for concrete slabs can have a significant effect on evaluating the safety and therefore should not be ignored in cases of high importance.
\end{abstract}

Keywords: Fire; specific heat; thermal conductivity; uncertainty; failure probability

\section{INTRODUCTION}

Fire is a rare event that can have a significant negative impact on people's safety. Luckily many fires are extinguished or suppressed early on by the occupants of the building or active measures (e.g. sprinklers). However, when fires progress beyond this level, they can cause serious damage to the structure. The effects of these, often referred to as 'structurally significant', fires are analysed in structural fire engineering (SFE). The main task of SFE is to produce a design solution that provides an adequate safety level, more precisely a solution with the failure probability below an explicit or implicit acceptable value.

\footnotetext{
${ }^{1}$ Corresponding author, Ghent University

e-mail: balsa.jovanovic@ugent.be, ORCID: https://orcid.org/0000-0001-5200-5848

${ }^{2}$ University at Buffalo

e-mail: negarkho@buffalo.edu, ORCID: https://orcid.org/0000-0003-3228-0097

${ }^{3}$ Ghent University

e-mail: thomas.thienpont @ ugent.be, ORCID: https://orcid.org/0000-0003-1466-3377

${ }^{4}$ Ghent University

e-mail: ranjitkumar.chaudhary@ ugent.be, ORCID: https://orcid.org/0000-0002-1104-5859

5 Ghent University

e-mail: ruben.vancoile@ugent.be, ORCID: https://orcid.org/0000-0002-9715-6786
}

https://doi.org/10.14264/363ff91 
In most design situations, the combination of prescribed fire resistance ratings and standardized calculation methods is sufficient to prove that the structure has an adequately low, although unquantified, failure probability. In special cases of high importance, however, adequate safety needs to be demonstrated explicitly. In those situations, (semi-)probabilistic calculations are required which directly take into account the stochastic nature of the input parameters. Even though the appropriate probabilistic models for some of these input variables are well-defined, others are often regarded as deterministic due to the lack of the appropriate models.

Probabilistic structural fire engineering (PSFE) is traditionally conducted using a large number of calculations with varying parameters and using numerical simulations. As the probabilistic approach is much more developed in classical structural engineering, the stochastic description of a large number of input variables needed for the structural analysis is well defined. This involves the geometry [1], loads [2] and material strength at ambient and elevated temperatures [3]. However, concerning the heat transfer part of the calculation, there is not much information available on the stochastic description of needed inputs.

Heat transfer has three modes: convection, radiation, and conduction. When conducting the heat transfer analysis for the temperature within structural members, the first two modes can be considered as a boundary condition and the last, conduction, as the main mode of temperature propagation through the concrete element. Conduction represents the diffusion of thermal energy (heat) within the material.

Thermal conductivity, $k$ describes the material's ability to conduct heat, and specific heat, $c_{p}$ represents the amount of energy needed to heat up a unit mass of the material by a unit temperature. Both of these properties are highly material and temperature dependant. Their influence on the thermal gradient within an element is defined by Fourier's law of (1D) conduction:

$$
\frac{\partial T}{\partial t}=\frac{k}{\rho c_{p}} \nabla^{2} T
$$

where $\frac{\partial T}{\partial t}$ represents the change of temperature with time, $\boldsymbol{k}\left[\frac{\boldsymbol{W}}{\boldsymbol{m} \cdot \boldsymbol{K}}\right]$ is the thermal conductivity, $\boldsymbol{c}_{\boldsymbol{p}}\left[\frac{\boldsymbol{J}}{\boldsymbol{k g} \cdot \boldsymbol{K}}\right]$ is the specific heat and $\rho$ is the material density.

The heat transfer analysis in SFE in its essence represents the numerical solution of the multidimensional version of equation (1). The most commonly used approach is to use the deterministic models for these thermal properties as provided in guidance documents. For concrete, some of the most widely used models are those presented in Eurocode [4]. Thermal conductivity is defined by two limits (upper and lower) that are given as quadratic functions of the temperature $T$ :

$$
\begin{array}{ll}
k_{\text {upper }}=2.00-0.2451 \cdot(T / 100)+0.0107 \cdot(T / 100)^{2} & \text { for } 20^{\circ} \mathrm{C} \leq T \leq 1200^{\circ} \mathrm{C} \\
k_{\text {lower }}=1.36-0.1360 \cdot(T / 100)+0.0057 \cdot(T / 100)^{2} & \text { for } 20^{\circ} \mathrm{C} \leq T \leq 1200^{\circ} \mathrm{C}
\end{array}
$$

where the choice of the exact value between these two limits is left to the user, but the lower values are recommended

On the other hand, specific heat is given as a single piecewise function:

$$
\begin{aligned}
& c_{p}=0.9 \\
& c_{p}=0.9+(T-100) / 1000 \\
& c_{p}=1.0+(T-200) / 2000 \\
& c_{p}=1.1
\end{aligned}
$$

$$
\begin{aligned}
& \text { for } 20^{\circ} \mathrm{C} \leq T \leq 100^{\circ} \mathrm{C} \\
& \text { for } 100^{\circ} \mathrm{C} \leq \mathrm{T} \leq 200^{\circ} \mathrm{C} \\
& \text { for } 200^{\circ} \mathrm{C} \leq T \leq 400^{\circ} \mathrm{C} \\
& \text { for } 400^{\circ} \mathrm{C} \leq T \leq 1200^{\circ} \mathrm{C}
\end{aligned}
$$


Both of these definitions are graphically presented in Figure 1.

Review of the available literature has shown that a significant scatter exists in the values of these thermal properties of concrete. In order to conduct a valid probabilistic calculation of the behaviour of a concrete structure exposed to fire, the uncertainty in the heat transfer analysis should be considered. In doing so, probabilistic models for the thermal properties of the material (i.e., thermal conductivity and specific heat) are needed.
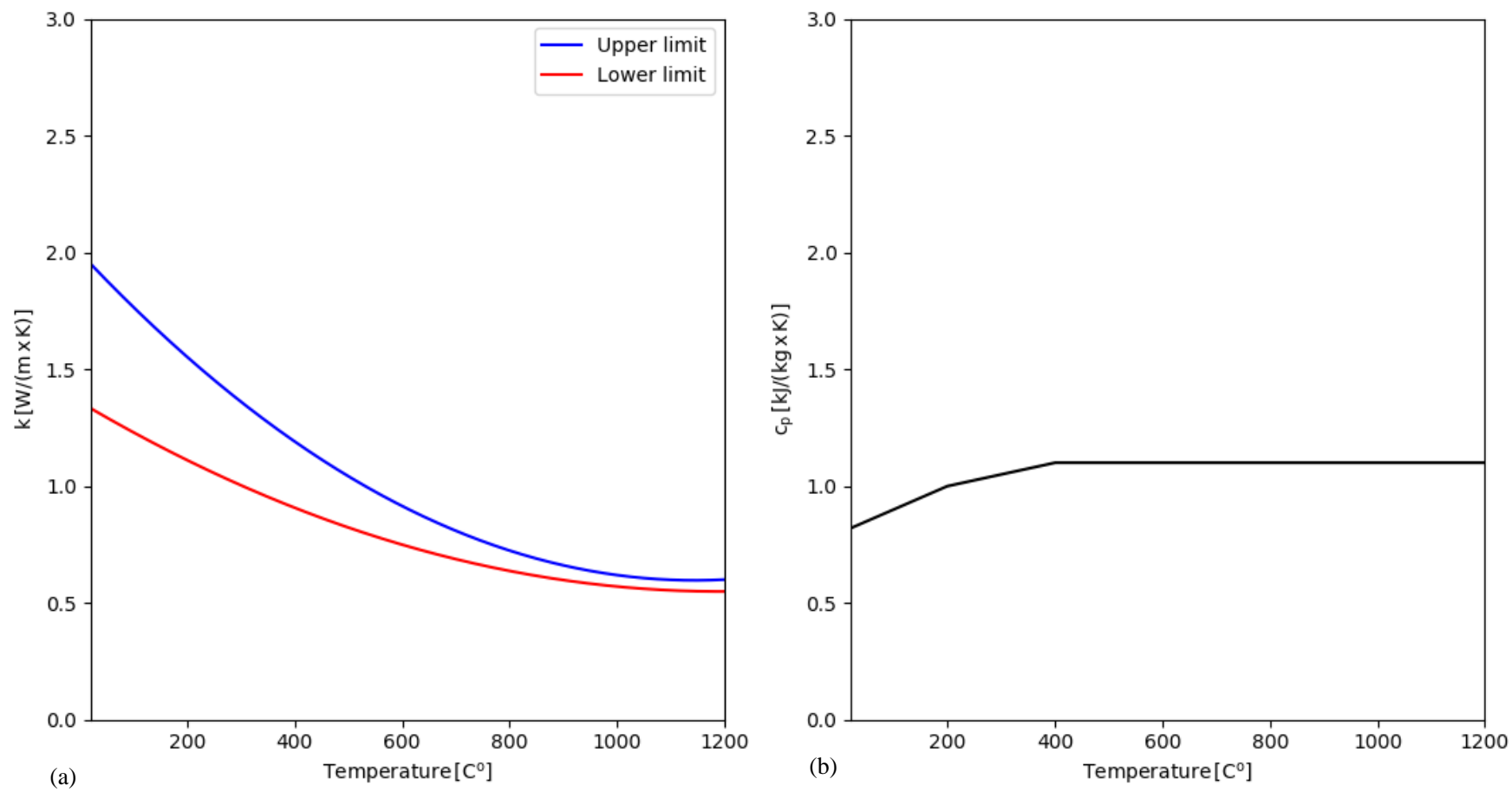

Figure 1. a) thermal conductivity $k$ and b) specific heat $c_{p}$ of concrete as a function of temperature as defined by the Eurocode [4]

\section{METHODOLOGY}

Probabilistic models in this study are developed with the intention of being used in numerical simulations. For that purpose, (i) the models have to be continuous functions across the appropriate temperature range, and (ii) there should be a closed-form function for each specific quantile value to facilitate implementation in the numerical simulations. The methodology for the model development is based on the one presented by Qureshi et al. [3], but with few modifications that are highlighted in the further text.

To obtain a closed-form function for each specific quantile value, the thermal conductivity and the specific heat are each individually considered to be described by the same probability distribution type (e.g. normal distribution, lognormal distribution) at any fixed temperature. The parameters that govern the probability distribution at a given temperature are then considered to be a continuous function of temperature. To obtain those parameters the first step is to organize the data in bins. More precisely, all the data points in the relatively narrow range around chosen temperature values are grouped into one bin and the mean and standard deviation of each bin are calculated. This way the closed-form equation for the mean and standard deviation as a function of temperature can be obtained by fitting a polynomial curve to these values. In order to take into account the different number of data points inside each bin and to capture the different amount of information each bin carries, each mean and standard deviation value is weighted with a number proportional to its size in the fitting process. 
After both the mean value and standard deviation are fitted in the form of a polynomial function, the appropriate probability distribution must be determined. This is done based on the Akaike Information Criteria (AIC) [5]:

$A I C=2 m-2 \ln (L)$

where $m$ represents the number of estimated parameters in the model and $L$ is the likelihood of the model for the given data. It must be noted that the AIC value can only be used as a comparative measure, more precisely, a single absolute value of AIC on its own does not contain any useful information. It can only be used to compare two or more models in a way that the model with the lowest AIC value represents the best fit.

The procedure to find the most appropriate distribution is as follows: Firstly, for each data point, the corresponding model mean and the standard deviation are calculated, based on the data point's temperature and the previously fitted polynomial functions. Based on those two values a specific theoretical distribution is defined and the likelihood of the data point is calculated. Lastly, the total likelihood is calculated by multiplying the values for each data point, and the AIC for a specific theoretical distribution is then obtained through (5). Using all the AIC values the best distribution is chosen.

It should be highlighted that even though the methodology described here is based on the work presented in [3] it has a couple of distinctive differences. First, the choice of adequate theoretical distribution is done more holistically, using the AIC for every data point based on the fitted curves for the governing parameters, instead of fitting the distribution for each bin separately, summing the AIC for each bin and then fitting a curve on the parameters. As its direct consequence, the second difference is fitting of the polynomial functions on mean and standard deviation values instead of the specific chosen distribution's parameters. The main advantage of this modified approach is that the best candidate distribution is chosen based on the final model and all the data points instead of only the data in the bins.

\section{RESULTS AND DISCUSSION}

The temperature range of $20-1200^{\circ} \mathrm{C}$ is chosen based on three factors, firstly the available data for constructing the model, secondly the fact that damage to the concrete at temperatures higher than $1000^{\circ} \mathrm{C}$ is large enough that it loses all of its mechanical properties, and finally the range of the commonly used Eurocode models.

Based on the available literature data, it is evident that the thermal properties differ significantly based on the type of concrete, whether its lightweight, normal strength (NSC) or high strength concrete (HSC). The focus in this paper is on the NSC as it is the most commonly used type. When considering the type of aggregate used in the concrete, it is decided to not make the distinction between siliceous and calcareous, in order to be in sync with the widely used Eurocode models for thermal properties [4].

Data needed for the creation of the models have been collected from the literature. Some data was found as tabulated values, but the majority had to be extrapolated from figures. The thermal conductivity dataset collected in this study consists of 382 points taken from 12 sources [6-17]. On the other hand, the specific heat data consists of 343 points taken from 9 sources $[6,8,9,12,14-16,18,19]$. 


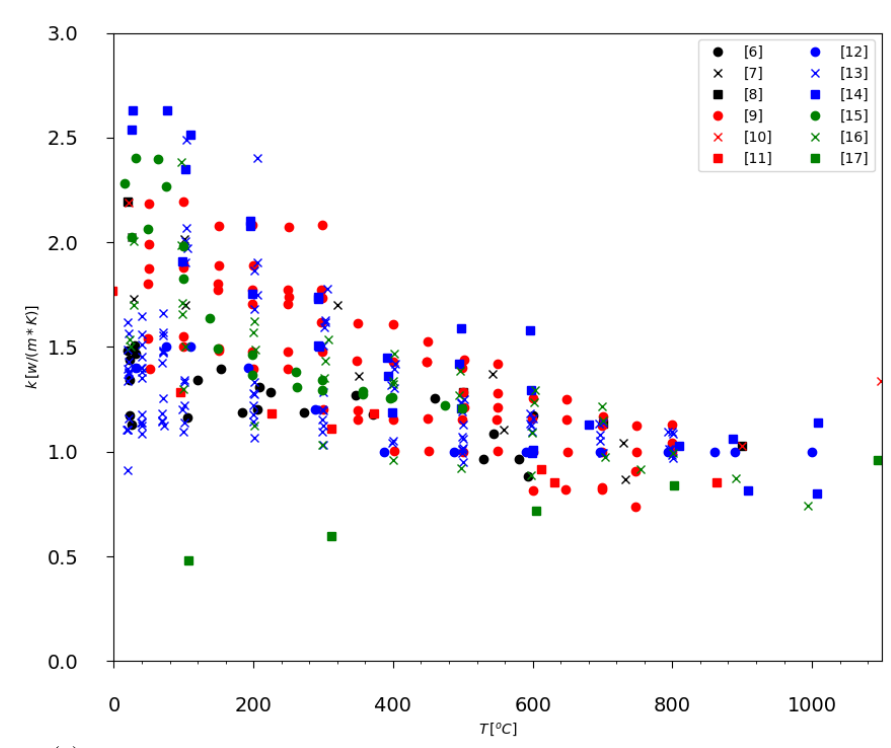

(a)

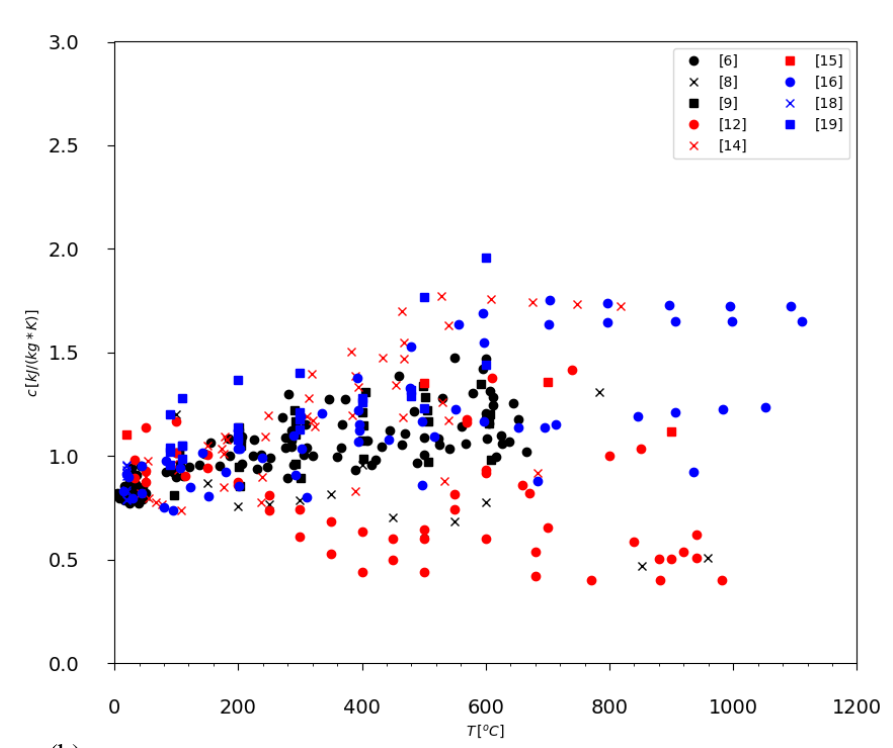

(b)

Figure 2. Acquired data points for a) thermal conductivity $k$ and b) specific heat $c_{p}$ of concrete at elevated temperature

From Figure 2, which presents all the acquired data points for both thermal conductivity and specific heat, it is evident that there is a large scatter, which implies considerable uncertainty. It is also clear that almost all of the data points are measured at a temperature that is close to a multiple of $50^{\circ} \mathrm{C}$. Thus, the data is grouped in bins with $50^{\circ} \pm 5^{\circ} \mathrm{C}$ temperature intervals. A minimum number of 5 data points are considered to form a bin (for example, there were only 4 points in the $350 \pm 5^{\circ} \mathrm{C}$ range for specific heat and therefore that bin was omitted). This concept is illustrated using a box plot in Figure 3, where it can also be observed that the lack of data at higher temperatures implies that no bins are created at temperatures higher than $800^{\circ} \mathrm{C}$ and $700^{\circ} \mathrm{C}$ for thermal conductivity and specific heat respectively.

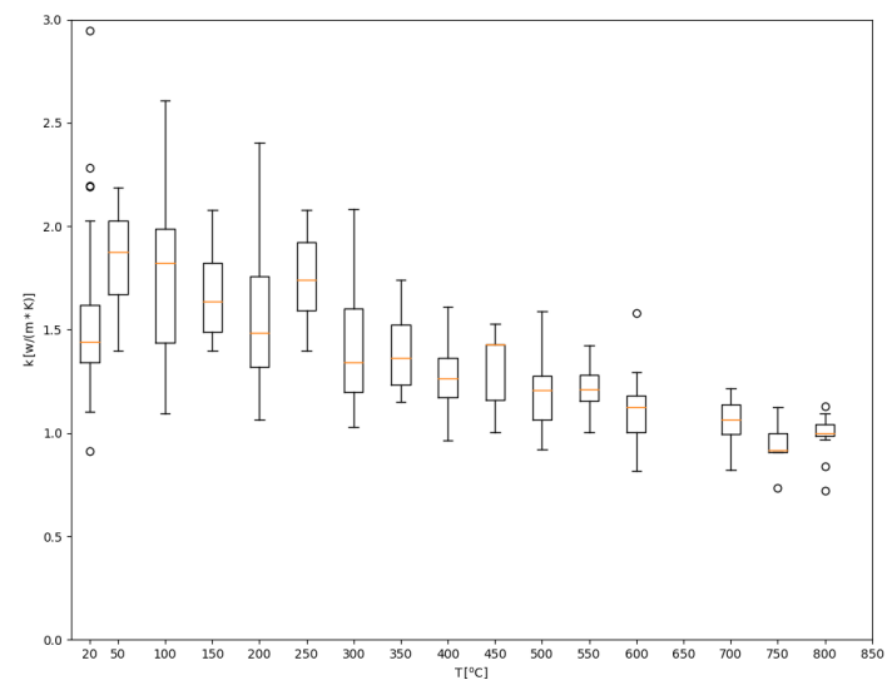

(a)

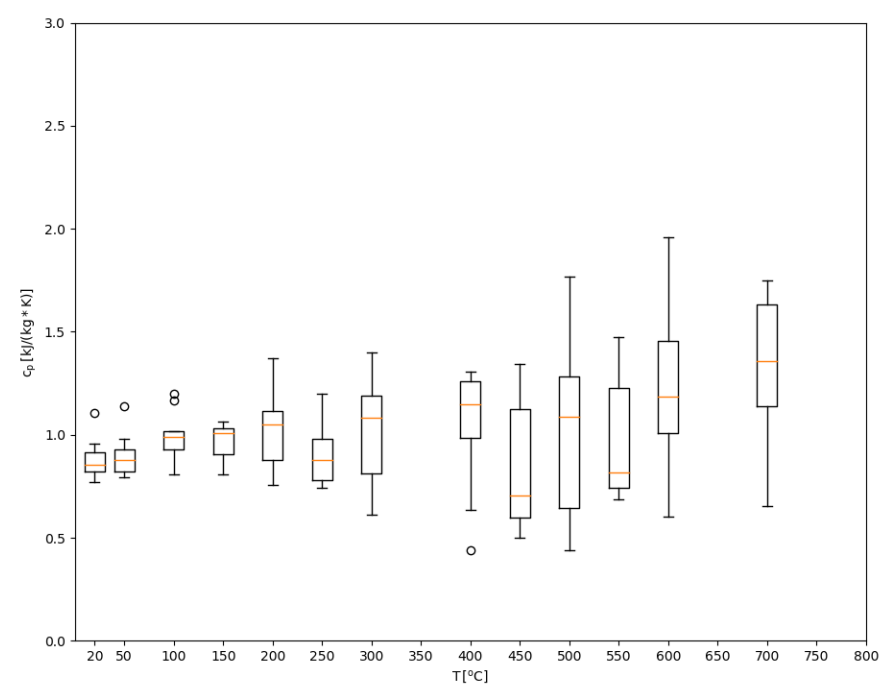

(b)

Figure 3. Box plot representing the variation of the a) thermal conductivity $k$ and b) specific heat $c_{p}$ of concrete data inside the bins

The fitting of the mean and standard deviation of the bins described earlier shows that the second order polynomial function is an appropriate fit. Because of the lack of data at temperatures higher than $800{ }^{\circ} \mathrm{C}$, one condition is added to the fitting process. Namely, in the case of the thermal conductivity, the functions are defined as monotonically decreasing in the range $20-1200{ }^{\circ} \mathrm{C}$ based on the global trends of the data and physical considerations. Similarly, the functions for the specific heat are considered as monotonically 
increasing in the same domain. The resulting equations are graphically presented in Figure 4 and are the following:

$$
\begin{aligned}
& \text { mean }_{k}=6.627 \times 10^{-7} \times T^{2}-1.458 \times 10^{-3} \times T+1.772 \\
& \text { std }_{k}=3.139 \times 10^{-7} \times T^{2}-0.691 \times 10^{-3} \times T+0.434 \\
& \text { mean }_{c_{p}}=-2.953 \times 10^{-7} \times T^{2}+6.498 \times 10^{-4} \times T+0.872 \\
& \text { std }_{c_{p}}=-3.500 \times 10^{-7} \times T^{2}+7.700 \times 10^{-4} \times T+0.042
\end{aligned}
$$

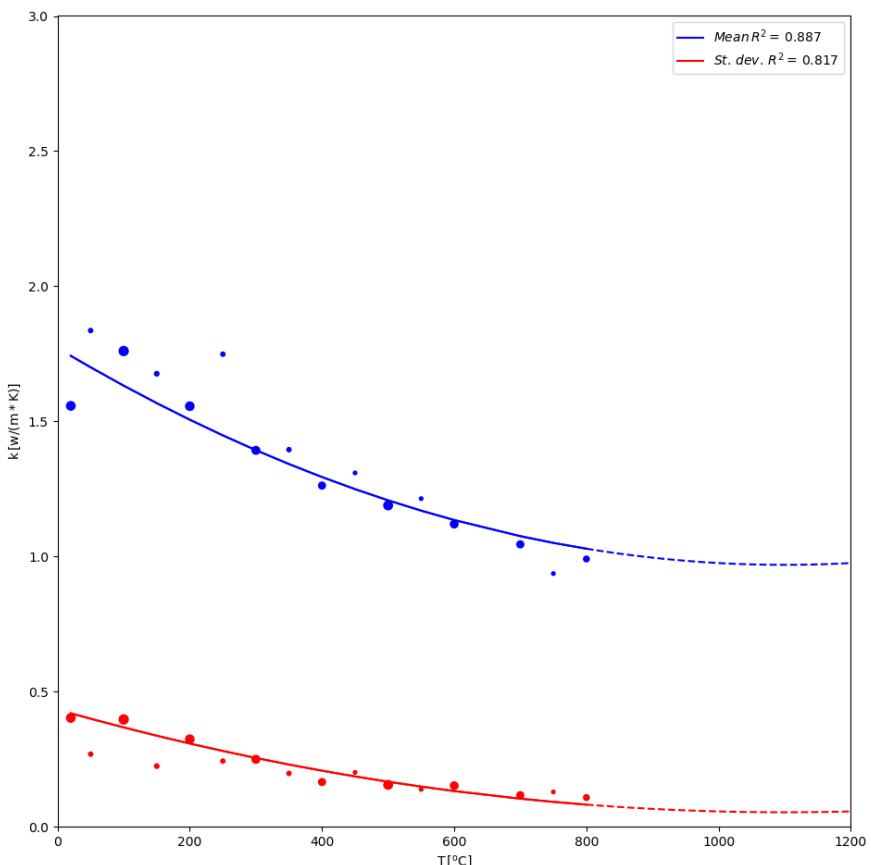

(a)

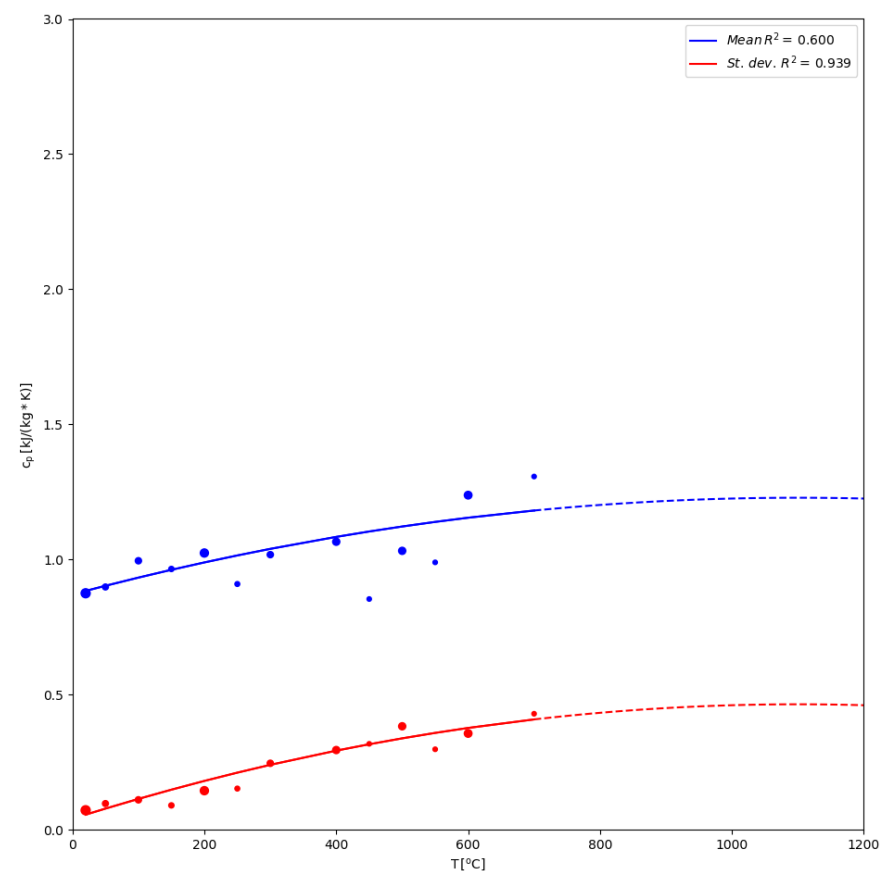

(b)

Figure 4. Polynomial function fit for the mean and standard deviation values for a) thermal conductivity $k$ and b) specific heat $c_{p}$ of concrete

Numerous two parameter theoretical distributions have been fitted in order to find the most adequate one for the models. More precisely, the fitted distributions were: Normal, Lognormal, Gamma, Gumbel, Beta44, Weibull, Inverse Gaussian, Logistic, Log-logistic, Rican, Nakagami and Birnbaum Saunders. Figure 5 shows the AIC values obtained for different distributions (for clarity only the five best fits are presented, as the values for the others were an order of magnitude larger). Both in the case of thermal conductivity and specific heat, the best fits (i.e., the lowest AIC score) are obtained by Normal and Gamma distributions. From the two, the Gamma distribution is chosen given that, on the contrary to the Normal distribution, it has zero probability for negative values (which are theoretically impossible for these thermal properties).

Figure 6 presents the obtained models alongside with the data points and Eurocode models. In the case of the specific heat, it can be seen that the $50 \%$ quantile values are close to those prescribed in Eurocode, however, that is not the case for thermal conductivity where the overlap between the two is minimal, especially at higher temperatures. Also, it can be seen that even though the data points for temperatures higher than $800^{\circ} \mathrm{C}$ were not used for the fiting of polygonal functions due to the lack of data, the overall model behaviour describes them relatively well. 


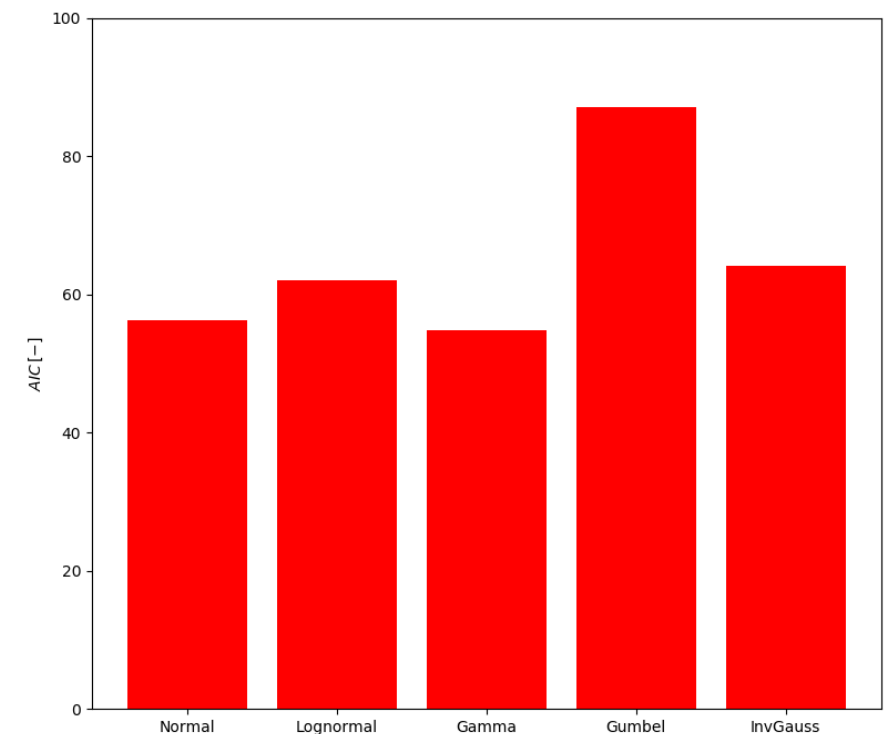

(a)

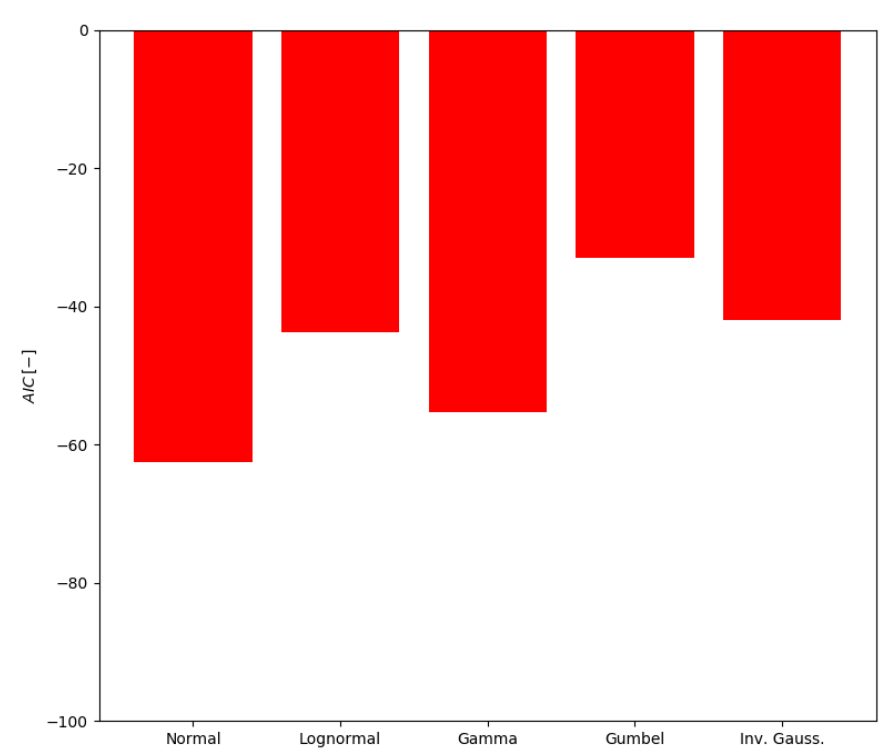

(b)

Figure 5. Akaike information criteria (AIC) comparison of a few theoretical distributions for a) thermal conductivity and b) specific heat of concrete

For the specific heat, the Eurocode [4] recommends an additional peak between $100^{\circ} \mathrm{C}$ and $200^{\circ} \mathrm{C}$, as a function of the moisture content, to take into account the moisture inside the concrete and the needed energy for moisture evaporation. Due to the great similarities of the $50 \%$ quantile curve, the same approach with the same values is recommended when using the probabilistic model for the specific heat of concrete. More precisely, the same moisture peak values are added to the specific heat curve for the desired quantile value. Even though the models do not provide a direct closed-form equation for any quantile value, the needed value can be easily calculated in two steps. Firstly the mean and the standard deviation are calculated for a given temperature and then based on the two calculated values and the desired quantile, the thermal property is evaluated using the well-known equations defining the Gamma distribution.
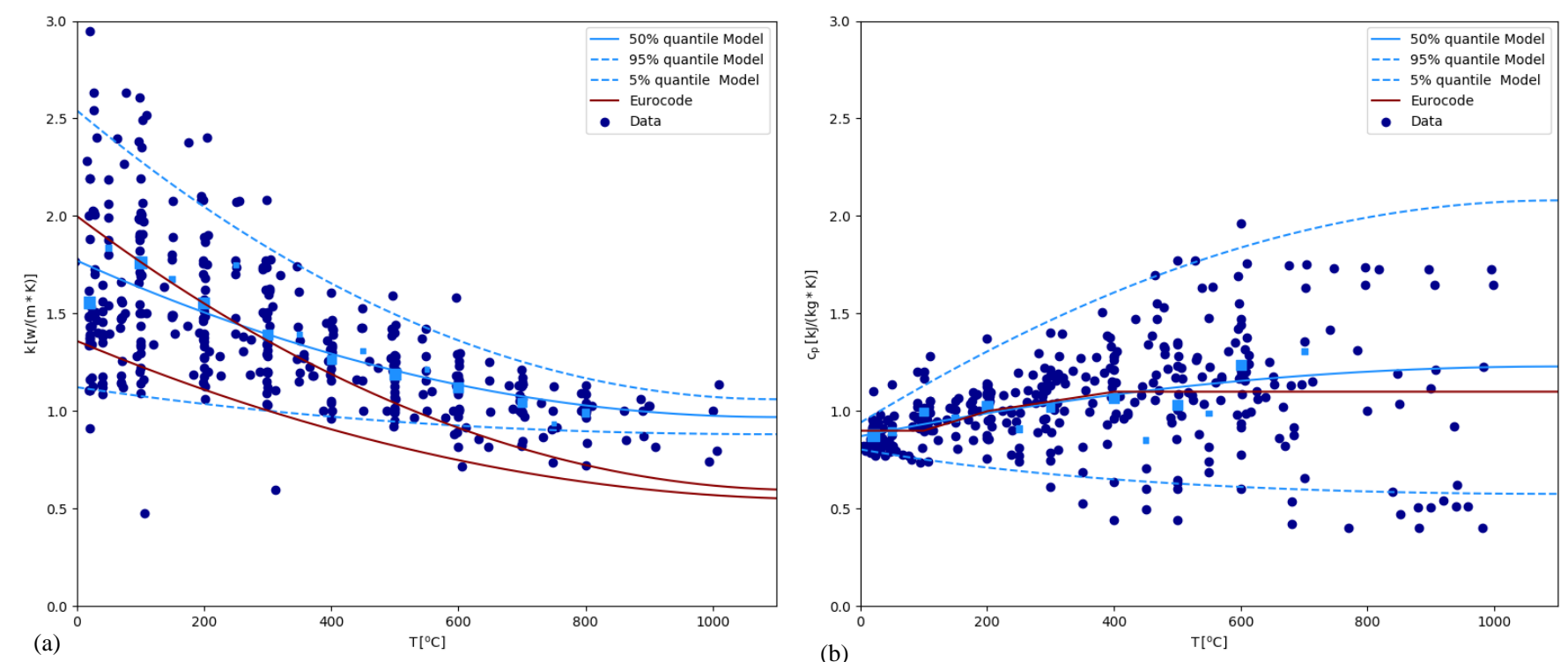

Figure 6. Probabilistic models for (a) thermal conductivity and (b) specific heat of concrete at elevated temperatures: $5 \%$, $50 \%$, and $95 \%$ quantiles, together with the model from Eurocode and the raw data.

\section{EXAMPLE}

An example of a simply supported concrete slab exposed to the standard fire will be used to demonstrate the influence of stochasticity in concrete thermal properties on the probability of failure. This example, but 
with deterministic thermal properties, in accordance with the Eurocode, was used in [20]. The slab in question is exposed to the fire only on the bottom side, while the top side is subjected to the ambient conditions. Thus, for the considered slab thickness, it is safe to assume that the temperatures on the top (i.e., the compression side) will not achieve values high enough to cause considerable strength reduction. For those reasons, it is justifiable to use the following equation to calculate the resisting moment of the slab, $M_{R}$ :

$M_{R}=k_{f y} \cdot f_{y, 20} \cdot A_{S} \cdot(h-\emptyset / 2-c)-\frac{\left(k_{f y} \cdot f_{y, 20} \cdot A_{S}\right)^{2}}{2 \cdot b \cdot f_{c}}$

where $b$ is the slab width (taken as $1 \mathrm{~m}$ ) and all the other parameters, along with their distributions, are presented in Table 1.

A probabilistic analysis based on $10^{4}$ Latin Hypercube Samples (LHS) is conducted in three parts: (i) first the heat transfer analysis using finite difference method and newly presented probabilistic models for thermal conductivity and specific heat are conducted in order to obtain the temperature distribution inside the slab; (ii) next, taking into account the temperature of the reinforcements inside the slab and Eqn. (10), the moment capacity of the slab is calculated; and (iii) finally, the probability of failure is calculated based on the loads and model uncertainties presented in Table 2 and using the limit state equation:

$Z=R-E=K_{R} \cdot M_{R}-K_{E} \cdot(G+Q)$

Figure 7 (a) shows the results of the heat transfer analysis, specifically the temperature gradient inside the slab after 120 and 180 minutes of standard fire exposure. In the figure, also the temperature gradient obtained using the Eurocode equations (lower limit) for thermal properties is presented. The most notable observation is that the temperatures are higher when calculated using the probabilistic models. The Eurocode temperature gradient is on the level of the 5\% quantile of the probabilistic model for both fire exposure times, and only close to the top surface of the slab, where the temperature is close to ambient condition, does the Eurocode temperature gradient come closer to the median results obtained with the probabilistic model.

Table 1. Stochastic input parameters used for the moment capacity calculation

\begin{tabular}{|c|c|c|c|c|}
\hline Parameter & Distribution & Mean $\mu$ & Standard deviation $\sigma$ & Ref \\
\hline $20^{\circ} \mathrm{C}$ concrete compressive strength, $f_{c, 20}[\mathrm{MPa}]$ & Lognormal & $42.9\left(f_{c k}=30 M P a\right)$ & 6.44 & {$[21]$} \\
\hline $20^{\circ} \mathrm{C}$ reinforcement yield strength, $f_{y, 20}[\mathrm{MPa}]$ & - & $560\left(f_{y k}=500 M P a\right)$ & - & - \\
\hline Steel yield stress retention factor, $k_{f y}[-]$ & Lognormal & Temperature dependent & Temperature dependent & {$[3]$} \\
\hline Reinforcement area, $A_{S}\left[\mathrm{~mm}^{2} / \mathrm{m}\right]$ & Normal & 785 & 16 & {$[21]$} \\
\hline Reinforcement diameter, $\emptyset[\mathrm{mm}]$ & - & 10 & - & - \\
\hline Slab height, $h[m]$ & - & 0.2 & - & - \\
\hline Concrete cover, $c[\mathrm{~mm}]$ & $\operatorname{Beta}[\mu \pm 3 \sigma]$ & $30+5=35$ & 5 & [1] \\
\hline
\end{tabular}

Figure 7 (b) shows the Probability Density Function (PDF) of moment capacity of the slab. It can be seen that an analysis using the Eurocode thermal properties overestimates the slab capacity compared to an analysis using the probabilistic models for thermal properties. This indeed can be explained by the higher temperatures in the reinforcement. The difference is even better illustrated in Figure 8, which shows the Cumulative Distribution Function (CDF) of the limit state from the Eqn. 11. The CDF value at $0 \mathrm{kNm}$ represents the probability of failure. Probabilites of failure in the case where the probabilistic model for the 
thermal properties is used are an order of magnitude higher compared to those obtained using the Eurocode thermal models, more precisely $2.82 \cdot 10^{-4}$ vs $2.37 \cdot 10^{-5}$ and $7.32 \cdot 10^{-2}$ vs $3.07 \cdot 10^{-3}$ for 120 and 180 min respectively. This difference in the probability of reaching the limit state illustrates how important it is to take into account the uncertainties in the thermal properties of concrete, especially since using the deterministic model is unconservative.
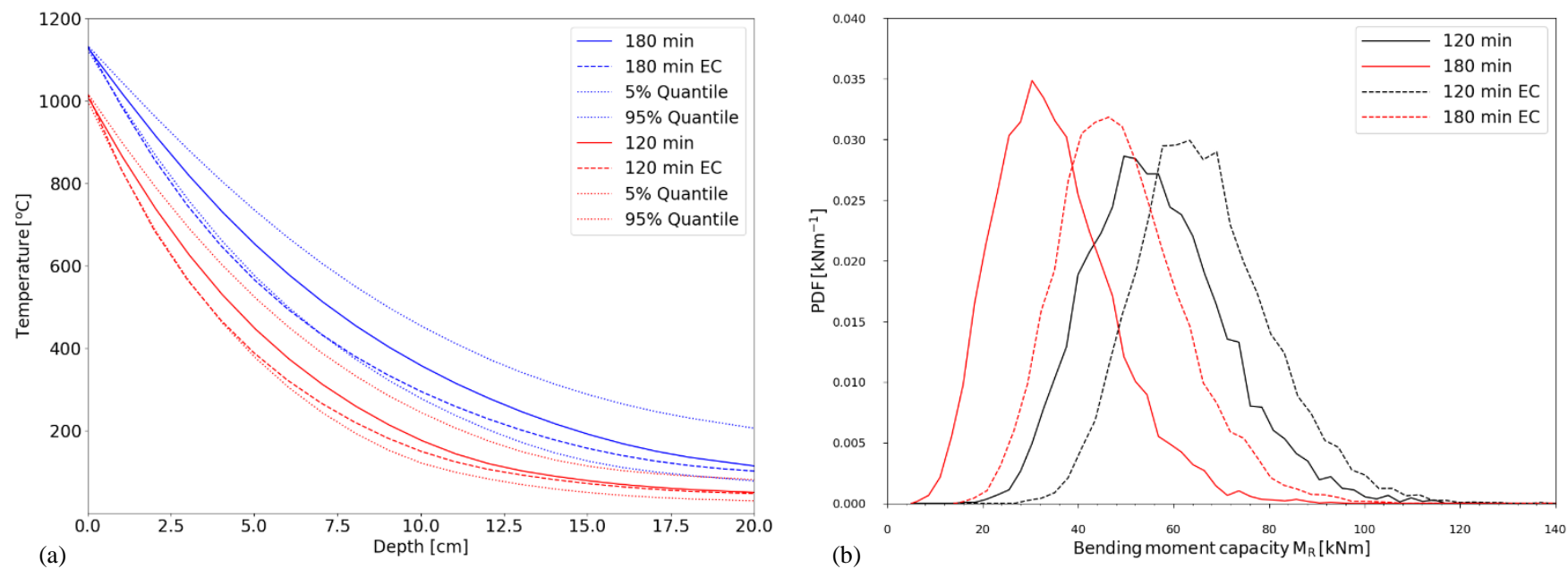

Figure 7. a) Temperature gradient inside of the concrete slab after 120 and 180 minutes of standard fire exposure at the bottom side, b) PDFs of the slab bending capacity calculated using the probabilistic and deterministic models for the thermal properties of concrete

Table 2. Stochastic input parameters used for calculation for the probability of failure

\begin{tabular}{lcccc}
\hline \hline Parameter & Distribution & Mean $\mu$ & Standard deviation $\sigma$ & Ref \\
\hline \hline Dead load, $G[\mathrm{kNm}]$ & Normal & 15 & 1.5 & {$[2]$} \\
\hline Live load, $\mathrm{Q}\left[\mathrm{kN} / \mathrm{m}^{2}\right]$ & Gamma & 1.875 & 1.78 & {$[2]$} \\
\hline Model uncertainty for the load effect, $K_{E}[-]$ & Lognormal & 1 & 0.1 & {$[2]$} \\
\hline Model uncertainty for the resistance effect, $K_{R}[-]$ & Lognormal & 1 & 0.15 & {$[2]$} \\
\hline
\end{tabular}

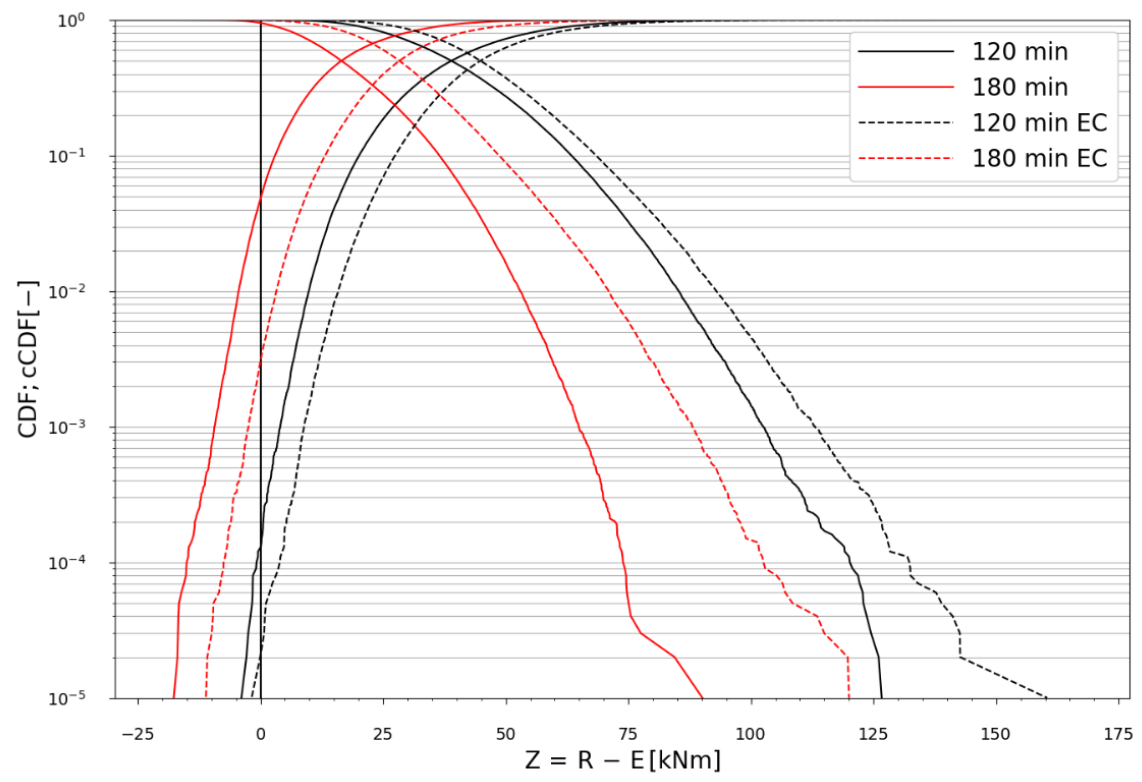

Figure $8 \mathrm{CDFs}$ of the limit state calculated using the probabilistic and deterministic models for the thermal properties of concrete 


\section{CONCLUSION}

Novel probabilistic models for thermal conductivity and specific heat of concrete are developed. The motivation for this study is the large uncertainty in the thermal properties of concrete that is evident from the large scatter in data reported in the literature. The models provide an efficient way to define the value of the thermal property at a specific temperature for the required quantile value, which is highly suitable for numerical simulations.

Models for both thermal parameters are defined using two steps. First, the mean and standard deviation are calculated for a specific temperature using Eqn. (6)-(9). Afterwards, the thermal property value can be generated based on the mean, standard deviation and the specified quantile value using the Gamma distribution.

In the case of the specific heat, the median, $50 \%$ quantile, values are similar to those prescribed in Eurocode, but the overall model still shows considerable variation. For thermal conductivity, however, the values are much larger and show a relatively small overlap with the Eurocode models. The influence of such differences is demonstrated using the probabilistic structural fire engineering analysis of a simply supported concrete slab. It was shown that a considerable difference in the temperature gradient, moment capacity and finally probability of failure could be expected when using the developed models as opposed to existing deterministic relationships.

Using the traditional deterministic models for thermal properties is justified for simple design situations, but the uncertainty of the thermal properties values cannot be neglected for special cases where the probabilistic calculations are needed, as traditional models could lead to unconservative results.

\section{REFERENCES}

1. JCSS, (2001). Probabilistic Model Code. Part 3.10 Dimensions. Joint Committee on Structural Safety

2. Jovanović, B, Van Coile, R, Hopkin, D, Elhami Khorasani, N, Lange, D, Gernay, T, (2020). Review of Current Practice in Probabilistic Structural Fire Engineering: Permanent and Live Load Modelling. Fire Technol. https://doi.org/10.1007/s10694-020-01005-w

3. Qureshi, R, Ni, S, Elhami Khorasani, N, Van Coile, R, Hopkin, D, Gernay, T, (2020). Probabilistic models for temperature dependent strength of steel and concrete. Journal of Structural Engineering 146:04020102. https://doi.org/10.1061/(asce)st.1943-541x.0002621

4. CEN, (2004). EN 1992-1-2:2004: Eurocode 2: Design of concrete structures - Part 1-2: General rules. Structural fire design. European Standard

5. Akaike, $\mathrm{H},(1974)$. A new look at the statistical model identification. IEEE Transactions on Automatic Control 19:716-723

6. Harmathy, T, Allen, L, (1973). Thermal properties of selected masonry unit concretes. Journal of The American Concrete Institute 70:132-142

7. Harada, T, Takeda, J, Yamane, S, Furumura, F, (1972). Strength, Elasticity and Thermal Properties of Concrete Subjected to Elevated Temperatures. ACI Structural Journal 34:377-406

8. Schneider, U, (1988). Concrete at high temperatures - A general review. Fire Safety Journal 13:55-68. https://doi.org/10.1016/0379-7112(88)90033-1

9. Adl-Zarrabi, B, Boström, L, Wickström, U, (2006). Using the TPS method for determining the thermal properties of concrete and wood at elevated temperature. Fire and Materials 30:359-369. https://doi.org/10.1002/fam.915

10. Banerjee, DK, (2016). An analytical approach for estimating uncertainty in measured temperatures of concrete slab during fire. Fire Safety Journal 82:30-36. https://doi.org/10.1016/j.firesaf.2016.03.005

11. Kodur, KR, Dwaikat, MMS, Dwaikat, MB, (2009). High-temperature properties of concrete for fire resistance modeling of structures. ACI Materials Journal 106:390

12. Shin, K-Y, Kim, S-B, Kim, J-H, Chung, M, Jung, P-S, (2002). Thermophysical Properties and Transient Heat Transfer of Concrete at elevated temperature. Nuclear Engineering and Design 212:233-241

13. Anderberg, Y, (1976). Fire-exposed concrete structures. An experimental and theoretical study. Bull Div 


\section{Struct Mech Concr Constr}

14. Lie, TT, Kodur, VKR, (1995). Thermal properties of fibre-reinforced concrete at elevated temperatures. National Research Council Canada, Institute for Research in Construction

15. Kakae, N, Miyamoto, K, Momma, T, Sawada, S, Kumagai, H, Ohga, Y, Hirai, H, Abiru, T, (2017). Physical and thermal properties of concrete subjected to high temperature. Journal of Advanced Concrete Technology 15:190-212. https://doi.org/10.3151/jact. 15.190

16. Hu, XF, Lie, TT, Polomark, GM, Maclaurin, JW, (1993). Thermal properties of building materials at elevated temperatures. Intern report/National Res Counc Canada, Inst Res Constr no 643

17. Marechal, J, (1972). Conductivity and Thermal Expansion Coefficients of Concrete as a Function of Temperature and Humidty. Special Publication 34:1047--1058

18. T.T. Lie, (1980). Structural Fire Protection

19. Naus, DJ, (2006). The effect of elevated temperature on concrete materials and structures-A literature review. Oak Ridge National Laboratory (United States)

20. Thienpont, T, Van Coile, R, De Corte, W, Caspeele, R, (2019). Determining a global resistance factor for simply supported fire exposed RC slabs. In: Concrete: innovations in materials, design and structures: fib Symposium 2019. pp 2191-2197

21. Holický, M, Sýkora, M, (2010). Stochastic models in analysis of structural reliability. In: International symposium on stochastic models in reliability engineering, life sciences and operation management, Beer Sheva, Israel 